\title{
Morphological Characteristics of Toxocara spp. Adults Determined by Electron Microscopy
}

\author{
lasmina Luca $^{*(\mathbb{D}}$, Yagoob Garedaghi $^{2}{ }^{(1)}$ \\ ${ }^{1}$ Department of Parasitology and Parasitic Diseases, Faculty of Veterinary Medicine, Banat University of Agricultural Sciences \\ and Veterinary Medicine, Timișoara, Romania \\ ${ }^{2}$ Department of Parasitology, Tabriz Branch, Islamic Azad University, Tabriz, Iran
}

\begin{abstract}
Ascarids are commonly diagnosed in dogs and cats. Toxocara canis and Toxocara cati eggs are similar, and the difference between the two species is based on the morphological characteristics of adult roundworms. Specific morphological components can be revealed by light microscopy, but a detailed description of the structural characteristics can be provided by electron microscopy. The body of adult roundworms dehydrates highly quickly, thus it is quite difficult to obtain clear images. The aim of this study was to provide morphological details specific to the Toxocara genus to complete the knowledge about the morphology of roundworms. The morphology of these nematodes can be thoroughly explained by certain phenomena related to their biological cycle and the preference for localization in various organs of the parasitic host.

Keywords: SEM, Ascarids, Carnivores
\end{abstract}

Received: February 2, 2021, Accepted: February 25, 2021, ePublished: April 1, 2021

\section{Introduction}

The genus Toxocara comprises 21 species, including Toxocara canis and Toxocara cati, which are the most common causes of larva migrans in humans (1).

Nematodes have a multi-layered cuticle, which is resistant to the action of gastric acid. It is generated by an underlying, smooth layer, that is called the hypodermis, whose nucleus is located in the four cords. The dorsal and ventral cords are composed of longitudinal axons. The lateral cord extends along the secretory canal and is covered by a series of apparently branched tubes. Somatic cells have convex, contractile areas, and a translucent nucleus with nucleoli is found in the cytoplasmic region (2).

At the level of the cuticle, there are four types of sensory papillae. In some species, the cervical papillae are located in the anterior part of the body and have various locations, shapes, and sizes. All these elements are used in highlighting the taxonomic characteristics.

Among the sensory papillae, the anterior ones (amphids) have various mechanical, namely, involvement in the movement of nematodes through various structures (3), chemical, and optical actions. Phasmids are found in the posterior part of the nematodes' body. They receive various external stimuli and help the parasite find a favorable environment for development and survival (4).

Electron microscopy is a surface imaging technique that allows a deep view of a three-dimensional field. The standard equipment of the electron microscope uses an electron beam that interacts with the electrons on the surface of the sample. The position of the beam is combined with the detected signal to produce an image (5). This technique has greatly helped in clarifying the external morphology of nematodes (6) and became an important tool in their morphology and taxonomy. Significant contributions have already been made to nematode ecology, host-parasite relationships, and biological control, with reviews on this technique representing positive findings (7).

\section{Objectives}

This study aimed to provide some perspectives and details about the electron microscopy technique, which has been shown to be useful in classifying nematodes. The main components of the external morphology of the roundworms (T. canis and T. cati) of dogs and cats were highlighted based on the study purpose.

\section{Materials and Methods}

This study was performed in the research laboratories at the Department of Parasitology and Parasitic Diseases of the Faculty of Veterinary Medicine in Timisoara, Romania.

Approximately 30 adult roundworms from dogs and cats, recently dewormed, were used and processed (fixing, washing, and drying) according to the method described by Snyder (8) Morphological components were 
highlighted using the Hitachi TM3000 Tabletop electron microscope at 5 and $15 \mathrm{kV}$.

\section{Results and Discussion}

The roundworms have an oral cavity surrounded by three triangular-shaped lips (one dorsal and two subventral lips) in their anterior portion (Figures $1 \mathrm{C}-\mathrm{D}$ and 2C). Their inner surfaces are composed of unicuspid or bicuspid dentate ridges (Figure 1D). Using electron microscopy, Ayoub identified trilobate, folded lips in T. canis adults with 4-5 teeth on the inner edge. They may be responsible for superficial or deep damage to intestinal walls, which can cause secondary bacterial infections and intoxication over time due to increased permeability (9). The other roundworms of the genus Toxocara (T. genettae) have a variable number of denticles on the lips. The male has $90-$ 98 and 82-96 denticles at the level of dorsal and subventral lips, respectively. Regarding females, the identified values included 96-102 (dorsal lip) and 90-96 (subventral lip) denticles, respectively (10).

Sensory organs (amphids) are also present on the lips (Figures 1D and 2C). Each lip has an amphid. The same number of amphids, but also the presence of two types of papillae (subventral and externolateral) have been reported in the rodent roundworms of the Muridae family, that is called T. apodemi (11).

The presence of the cervical alae in the anterior part at T. canis and T. cati offers a characteristic aspect of "spear" (Figure 1C) and "arrowhead" (Figure 2A), respectively. Some studies indicated cervical alae width values of 0.19 $0.217 \mathrm{~mm}$ and $0.21-0.315 \mathrm{~mm}$ for T. canis and T. Cati, respectively (10). The cuticle has transverse striations (Figures $1 \mathrm{~A}-\mathrm{B}$ and $2 \mathrm{~B}$ ). The posterior end is curved in males (Figures $1 \mathrm{~F}$ and $2 \mathrm{D}$ ). The cloaca is surrounded by 32 pairs of sensory papillae (phasmids), including 26 precloacal (Figure 1A-B-F) and 4 postcloacal (Figure 1E) ones. In the case of T. canis, they have a circular shape, are convex, are well-contoured at the cuticular level, and

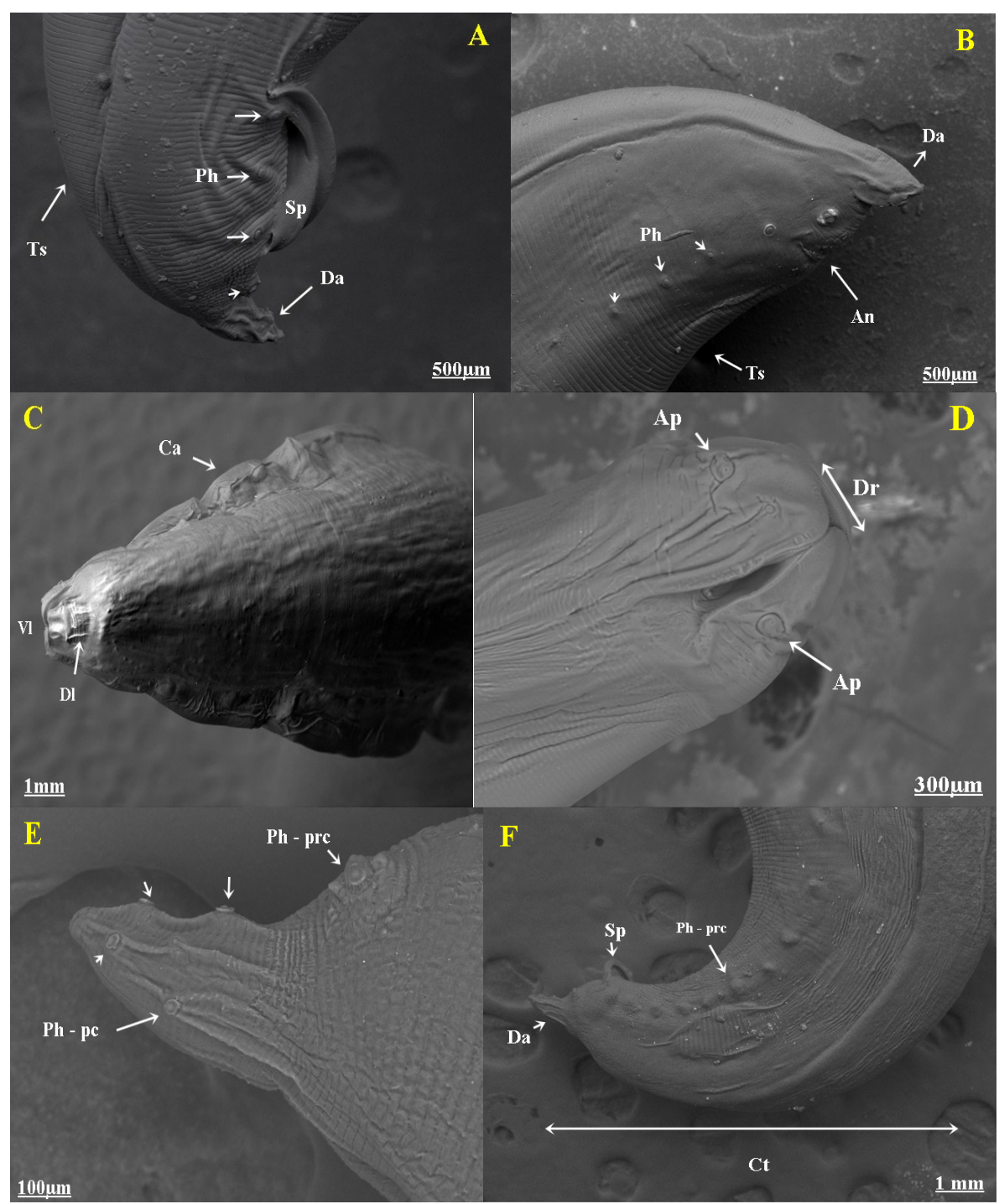

Figure 1. Toxocara canis: (A) Male Tail, (B) Female tail, (C) Head, (D) Mouth, (E) Posterior Part, and (F) Male Tail. Note. Da: Digitiform appendix; Ts: Transverse striations; Ph: Phasmids; Sp: Spicule; An: Anus; DI: Dorsal lip; VI: Ventral lips; Ca: Cervical alae; Ap: Amphid; Dr: Dentate ridges; Ph prc: Precloacal phasmids; $\mathrm{Ph}$ - pc: Postcloacal phasmids; Sp: Spicule; Ph - prc; Ct: Curved tail. 


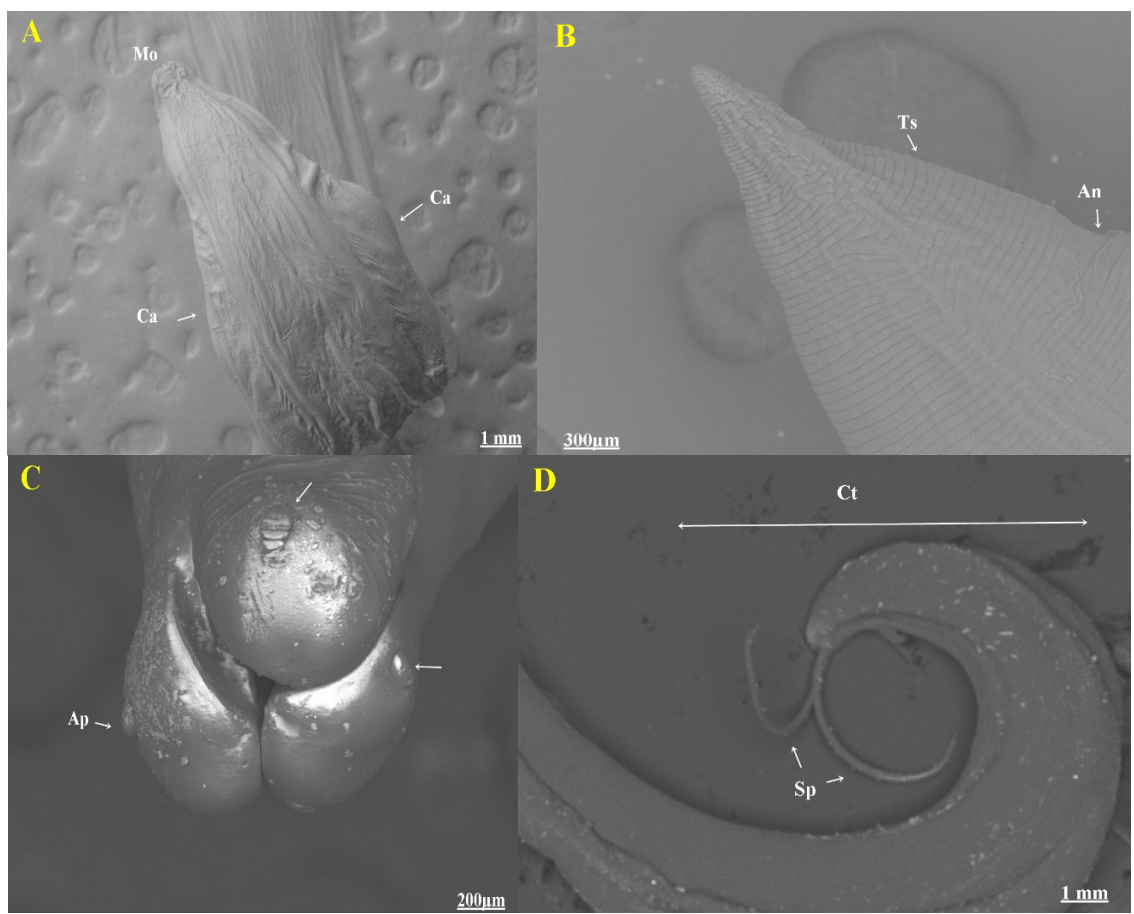

Figure 2. Toxocara cati: (A) Head, (B) Female Tail, (C) Mouth, and (D) Male posterior. Note. Mo: Mouth; Ca: Cervical alae; Ts: Transverse striations; An: Anus; Ap: Amphids; Sp: Spicules; Ct: Curved tail.

have a "button" appearance (Figure 1A-B-F). As regards $T$. cati, they are not easy to highlight, and the same number of postcloacal phasmids was reported in another electron microscopy study in adults of T. canis (9).

The digitiform appendix is present in the adults of $T$. canis (Figure 1A-B). In males, the spicules (Figures $1 \mathrm{~A}-\mathrm{F}$ and 2D) have different sizes and are uneven. Other researchers found that the left spicule is more developed in the middle and thinner at the end, and the right one is smaller and curved (9). According to the literature, the dimensions of the spicules are known as 1.71-1.90 mm and 0.91-0.97 for T. cati and T. canis, respectively $(11,12)$.

In females, the vulva is present in the anterior third of the body, and the circular anus (Figures 1B and 2B) can be observed in the region of the tail.

\section{Conflict of Interests}

The authors declare that they have no conflict of interests.

\section{Ethical Issues}

Not applicable.

\section{Funding}

Not applicable.

\section{References}

1. Rocha IML, Oréfice F, Soares IP, et al. Toxocaríase. In: Oréfice F, ed. Uveíte Clínica e Cirúrgica. 2nd ed. Rio de Janeiro: Cultura Médica; 2005.

2. Shalaby HA, Abdel-Shafy S, Abdel-Rahman KA, Derbala AA. Comparative in vitro effect of artemether and albendazole on adult Toxocara canis. Parasitol Res. 2009;105(4):967-976.

\section{doi:10.1007/s00436-009-1479-9}

3. Naem S. The comparative morphology of three equine habronematid nematodes: SEM observations. Parasitol Res. 2007;101(5):1303-1310. doi:10.1007/s00436-007-0637-1

4. McLaren DJ. Nematode sense organs. Adv Parasitol. 1976;14:195-265. doi:10.1016/s0065-308x(08)60515-1

5. Cheville NF, Stasko J. Techniques in electron microscopy of animal tissue. Vet Pathol. 2014;51(1):28-41. doi:10.1177/0300985813505114

6. Hirschmann H. Scanning electron microscopy as a tool in nematode taxonomy. In: Stone AR, Platt HM, Khalil LF, eds. Concepts in Nematode Systematics. New York: Academic Press; 1983:388.

7. Wergin WP. Scanning electron microscopic techniques and applications for use in nematology. In: Zuckerman BM, Rhode RA, eds. Plant Parasitic Nematodes. Vol III. New York: Academic Press; 1981:508.

8. Snyder DE. Scanning electron microscopy of adult Parascaris equorum (Nematoda). Proc Helminthol Soc Wash. 1985;52(2):237-243.

9. Ayoub MB. Parasitic infection in stray cats and dogs with special reference to ultrastructure of the recovered worms. Anim Health Res J. 2014;2(3):165-178.

10. Alexander U, Lim CW, Kim B, Hong EJ, Kim HC, Park BK. Morphological and molecular characterization of Toxocara tanuki (Nematoda: Ascaridae) from Korean raccoon dog, Nyctereutes procyonoides koreensis. Korean J Parasitol. 2018;56(6):567-575. doi:10.3347/kjp.2018.56.6.567

11. Garedaghi Y, Shabestari-AsI SA. Contamination rate of Toxocara spp. eggs in the public parks of Tabriz city: a short report. J Rafsanjan Univ Med Sci. 2012;11(2):173-178. [Persian].

12. Warren G. Studies on the morphology and taxonomy of the genera Toxocara Stiles, 1905 and Neoascaris Travassos, 1927. Zool Anz. 1970;185(5-6):393-442.

(C) 2021 The Author(s); This is an open-access article distributed under the terms of the Creative Commons Attribution License (http:// creativecommons.org/licenses/by/4.0), which permits unrestricted use, distribution, and reproduction in any medium, provided the original work is properly cited. 